\title{
ELECTROMYOGRAPHIC RESPONSE TO DISPLACEMENT OF DIFFERENT FORELIMB JOINTS IN THE SQUIRREL MONKEY ${ }^{1}$
}

\author{
F. A. LENZ, W. G. TATTON, ${ }^{2}$ and R. R. TASKER \\ Playfair Neuroscience Unit and Division of Neurosurgery, University of T'oronto, Toronto, Canada
}

Received July 29, 1982; Revised October 29, 1982; Accepted November 5, 1982

\begin{abstract}
The electromyographic (EMG) reflex response evoked in muscles stretched by imposed angular joint displacement has previously been studied in a variety of muscles and species. In most muscles studied, the EMG response consists of an initial burst of activity at latencies comparable to tendon tap responses followed by one or more bursts of EMG activity occurring at latencies less than somatosensory reaction time. The longer latency bursts or peaks of activity have often been assumed to be of similar origin in functionally diverse muscles. The present experiments were performed to examine the EMG response to imposed joint displacement in several different muscles of the squirrel monkey forelimb.

The EMG was studied in muscles stretched by elbow, wrist, and metacarpophalangeal joint extension. Early (M1) and long (M2) latency peaks of activity could be observed in the EMG responses of short head of biceps (SHB), flexor carpi ulnaris (FCU), and flexor digitorum profundus (FDP), at latencies which are similar to those reported in other primate species allowing for differences in the size of the animals. The intervals between these peaks did not correspond to the period of oscillations in tension which occurred after the onset of the joint extension. The M2 peak consistently occurred later in SHB than in FCU and FDP. The M1 peak was smaller in distal than in proximal muscles and was frequently absent in FDP at resting levels of EMG activity. The ratio of $\mathrm{M} 1$ to $\mathrm{M} 1+\mathrm{M} 2$ activity for different muscles revealed that proximal muscles had prominent early with less prominent long latency activity, whereas distal muscles had minimal early latency activity with prominent long latency activity. The onset of EMG activity approached tendon tap latencies in FCU at high base line EMG levels but in most cases occurred at approximately twice tendon tap latencies in FDP at high base line EMG levels. The results demonstrate the presence of significant differences between the EMG response to joint displacement in the proximal-distal series of forelimb flexors. Differences in the reflex response to joint displacement are discussed in view of twitch times and motor unit composition of the muscles studied.
\end{abstract}

In 1954, Hammond first reported a prolonged electromyographic (EMG) response in the human biceps to imposed elbow extension (Hammond, 1954). The EMG response was composed of a short latency burst followed by a silent period and then by a second burst of EMG activity. The second burst was prolonged if the subject had been instructed to "resist" and shortened if the

\footnotetext{
${ }^{1}$ We are indebted to R. Andrews and W. Bedingham who wrote the computer programs used in this study and to Dr. V. B. Brooks for his assistance with the interspecies comparisons. This work was supported by the Multiple Sclerosis Society of Canada, the Ontario Heart Foundation Grant Str-T-2, the Toronto General Hospital Foundation, and Medical Research Council of Canada Grant MA 5218. F. A. L. was a Medical Research Council of Canada Fellow.

${ }^{2}$ To whom correspondence should be addressed at Playfair Neuroscience Unit, Toronto Western Hospital, 399 Bathurst Street, Toronto, Canada M5T 2S8.
}

subject was instructed to "let go." This long latency activity was thought to be reflexive because when the subject was instructed to "let go" it occurred earlier than the shortest reaction time latencies (Hammond, 1960; Evarts and Vaughn, 1978). A resurgence of interest in Hammond's observation has occurred over the past decade with the application of torque motor technology to the study of motor control (see discussion in Colbourne and Evarts, 1978). Using this technique, short and long latency reflex activity has been reported for numerous different muscle groups and species. The EMG response can occur as three bursts of activity which were named M1, M2, and M3 by Tatton et al. (1975). Long latency activity has been reported in muscles acting across the human thumb (Marsden et al., 1976a), wrist (Lee and Tatton, 1975; O'Rian et al., 1978), elbow (Hammond, 1954; Evarts and Granit, 1976; Mortimer and Johnson, 
1976; Chan et al., 1979a), knee (Chan et al., 1979a), and ankle (Chan et al., 1979b; Gottlieb and Agarwal, 1979). Similar long latency responses have been observed in primate wrist (Bawa and Tatton, 1979; Tatton and Bawa, 1979) and elbow muscles (Tatton et al., 1975; Evarts and Tanji, 1976; Vilis and Cooke, 1976; Cooke and Eastman, 1977) and in cat triceps brachii (Ghez and Shinoda, 1978; North and Tatton, 1980).

Implicit in the conclusions of many of these studies is the assumption that similar peripheral and/or central mechanisms generate the later components of the EMG response in different muscle groups (Marsden et al., 1976b; Chan et al., 1979a) and in different species (Ghez and Shinoda, 1978). This latter assumption is partly based on the premise that, after taking size differences into account, latency measurements are comparable between primates and humans (Lee and Tatton, 1975, 1978; Tatton et al., 1979), between different primate species (Vilis and Cooke, 1976; Miller and Brooks, 1981), and between primates and cats (Ghez and Shinoda, 1978). However, this assumption has never been evaluated quantitatively, and the hazards of assuming that the results in one species are comparable to those obtained in other species have been discussed (Wiesendanger, 1978; Bawa and Tatton, 1979).

Even within one species, the assumption of a common mechanism of the long latency EMG response may not be valid in muscles of different function and composition. For example, the response of the intrinsic muscles of the hand and the gastrocnemius soleus complex to peripheral nerve stimulation is markedly different. In intrinsic hand muscles peripheral nerve stimulation produces early EMG activity followed directly by long latency activity (Upton et al., 1971; Milner-Brown et al., 1975; Stanley, 1978), whereas a silent period follows the early response in gastrocnemius (Magladery et al., 1951). Even between the flexors of the human elbow and phalangeal joints, significant differences are evident. The tension generated by the reflex response to imposed displacements occurs after a longer delay in muscles acting across the metacarpophalangeal joint than in those acting across the elbow (Brown et al., 1976; Rack et al., 1978). Hence, the nature of the EMG response in these different muscles bears further examination. In the present study, we have examined the EMG activity evoled in muscles stretched by displacement of several different forelimb joints in one primate species.

\section{Materials and Methods}

These studies were performed over a period of 18 months on six normal squirrel monkeys (Saimiri sciureus) weighing between 600 and $1000 \mathrm{gm}$. The muscles studied were short head of biceps (SHB), an elbow flexor, flexor carpi ulnaris (FCU), a wrist flexor, and flexor digitorum profundus (FDP), a finger flexor. Alert untrained monkeys were held in a primate chair under minimal restraint. EMG was recorded through pairs of Teflon-coated stainless steel wires (Basmajian and Stecko, 1962) which were inserted together into the muscle through a common needle. Electrode placement was monitored before and after each trial by observing the relationship of EMG activity to movements initiated by manipulation of different joints. When the electrode was positioned so that it responded selectively to movement of one joint, the arm proximal to the joint in question was immobilized in a padded plastic cast (Sans Splint, Smith and Nephew, Lachine, Quebec) fixed to the housing of the torque motor. The limb distal to the joint was splinted and secured to the handle of the torque motor. The cast was placed so that it did not impede rotation of the joint, which was co-axial with the shaft of the motor. All torque motor-imposed joint displacements were in the horizontal plane: the metacarpophalangeal (MCP) and wrist joints with the forearm positioned midway between the extremes of pronation and supination, the elbow joint with the shoulder abducted to $90^{\circ}$ (Fig. 1).

The handle of the torque motor to which the limb was attached was fixed to a metal rod fastened to the shaft of the torque motor (2015R, Torque Systems, Waltham, MA) along a radius from the axis of the motor. Joint angle was measured by a potentiometer linked to the shaft of the motor. The torque generated by resistance to the imposed movement was measured with high sensitivity strain gauges (N11-FA-10-1000-11, Showa Measuring Instruments Co., Tokyo) which were affixed immediately below the handle. The strain transducers were found to be linear within the range of $\pm 0.5 \mathrm{~N}$ (Newton). Tension oscillations following the onset of joint displacement can give rise to bursts of afferent activity, which may cause peaks of EMG activity (Hagbarth et al., 1981). In order to demonstrate that EMG activity could be independent of tension oscillations, the period of these oscillations was altered so that it did not correspond to the interval between EMG peaks. The high frequency and low amplitude of the tension oscillations (see below) was achieved by making the transducer very stiff and minimizing the mass of the handle distal to the transducer.

The torque motor was driven by square wave pulses of various amplitudes and of approximately $500-\mathrm{msec} \mathrm{du}-$ ration, which were presented in pseudorandom order and at random intervals. The torque loads were chosen so that displacements about all the different joints included a similar range of initial velocities and accelerations. The initial velocity and acceleration were calculated by analysis of the position recorded over the first $15 \mathrm{msec}$ following the onset of the square wave pulse to the torque motor. About the elbow, torque loads in the range of 0.05 to $0.30 \mathrm{Nm}$ (newton-meter) were imposed and produced initial velocities of 50 to $170^{\circ} / \mathrm{sec}$ and accelerations of 8,000 to $29,000^{\circ} / \mathrm{sec}^{2}$. Torque loads of 0.03 to $0.22 \mathrm{Nm}$ produced initial velocities of 40 to $270^{\circ} / \mathrm{sec}$ with accelerations of 6,000 to $37,000 \% / \mathrm{sec}^{2}$ about the wrist and velocities of 50 to $310^{\circ} / \mathrm{sec}$ with accelerations of 5,000 to $50,000^{\circ} / \mathrm{sec}^{2}$ about the MCP joint. The rotation of the torque motor shaft was limited by stops separated by 20 to $30^{\circ}$. The limb was automatically returned to a uniform starting position between trials. EMG, joint angle, and tension were recorded on magnetic tape along with a sequence of pulses which encoded the occurrence and magnitude of a torque pulse for later analysis. The data for the randomly ordered step load magnitudes were sorted and averaged off-line by a Digital PDP 11-34 computer. Averages were composed of 20 to 30 events in 


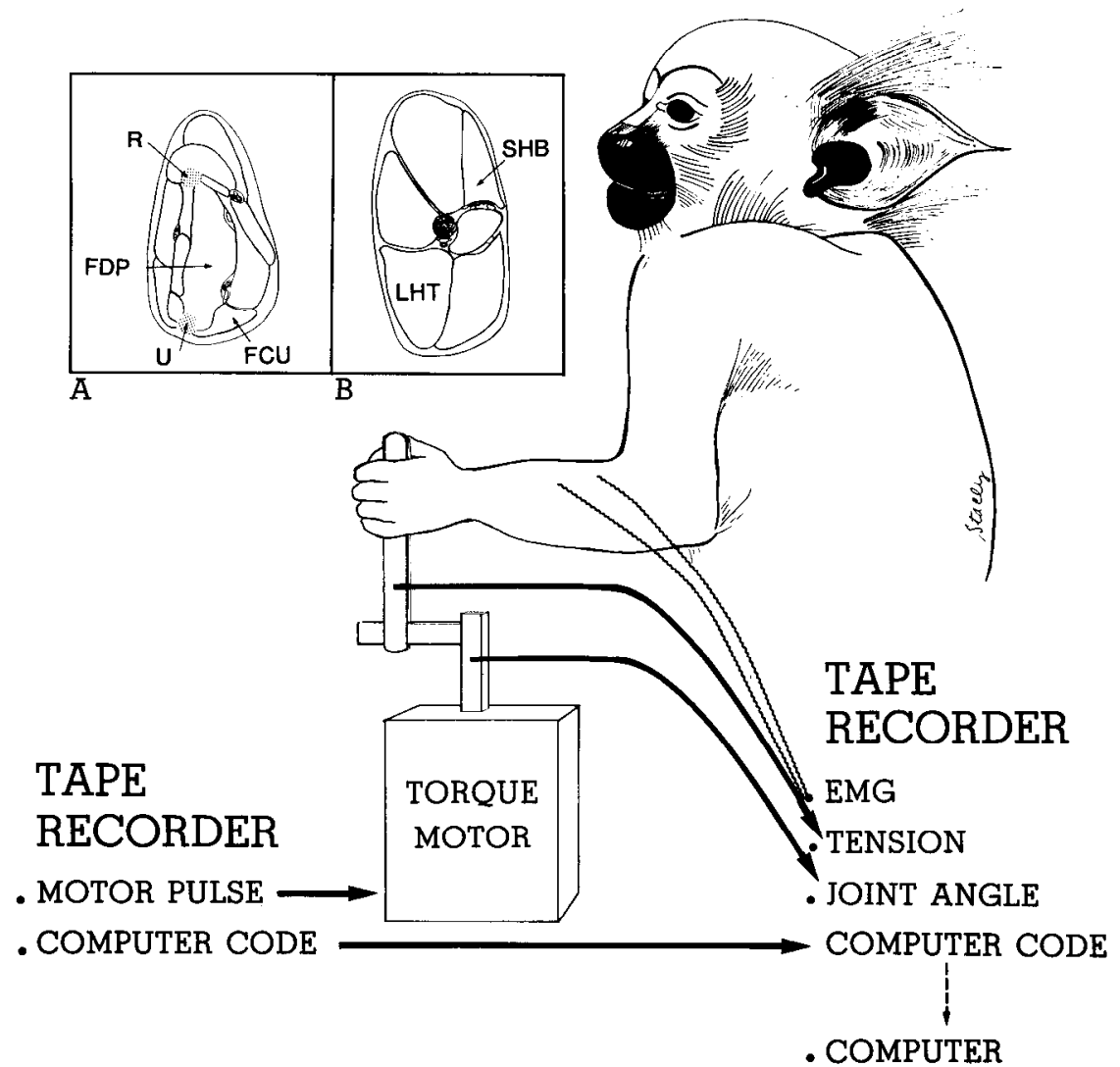

Figure 1. Schematic diagram of the experimental apparatus. Torque motor step loads driven by a square wave signal were used to impose angular displacements about the metacarpophalangeal, elbow, or wrist joint, as shown. A binary word (computer code) which was recorded on tape aided the sorting of the responses to the randomly ordered loads before averaging. Insets $A$ and $B$ are cross-sections of the forearm and arm showing the positions of the muscles studied. $R$, radius; $U$, ulna; LHT, lateral head of triceps.

which the following criteria were satisfied: (1) low background EMG levels (prior to the onset of the torque pulse) in agonist and antagonist muscles, and (2) baseline tensions of zero $\pm 10 \%$ of the tension generated at the onset of the highest torque load employed.

Whole muscle twitch contraction time to peak was measured for the finger flexors by stimulation of the ulnar and median nerves in the brachial bundle at the medial aspect of the humerus. For this procedure, the monkey was sedated with ketamine $(10 \mathrm{mg} / \mathrm{kg})$. The nerve was stimulated once every $4 \mathrm{sec}$, and the first 10 stimuli were averaged. Measurement of muscle tension by an external transducer in human adductor digiti minimi has been shown to give the same tension response latency as is measured by a transducer applied to the muscle tendon (Buchtal and Schmalbruch, 1970, 1980). Since we used a similar external tension transducer, we believe that this method gives a good estimate of whole muscle contraction time for FDP.

\section{Results}

Latency of onset of EMG responses. The EMG response to joint displacement in the muscles studied often showed a segmented pattern of two or three consecutive peaks of EMG activity which were termed M1, M2, and M3 (after Tatton et al., 1975). Figure $2 A$ shows the response in biceps to a torque load extending the elbow.
All averages are taken from the onset of the square wave signal driving the torque motor and hence include the isometric time constant of the torque motor, which we measured to be $1.8 \mathrm{msec}$. The top trace shows the force resisting the torque motor-imposed displacement of the forearm which is shown in the second trace. The third trace shows the full wave rectified biceps EMG. M3 activity was variably present in agreement with previous work (Lee and Tatton, 1975) and was not analyzed. Figure 2, $B$ and $C$ present data in the same format for FCU and FDP in response to wrist and MCP joint displacement, respectively. In these figures oscillations in tension are superimposed upon the step increase in torque following the square wave pulse to the torque motor at time zero. Examining the record for FCU (Fig. $2 B$ ) one can see tension peaks which occur at approximately the same interval as the consecutive EMG peaks. Therefore, it could be argued that the EMG bursts are caused by synchronizing bursts of afferent activity. This does not seem to be the case in FDP because there is no EMG activity corresponding to the first beat of the oscillation in the tension trace (Fig. $2 C$ ). The average from SHB (Fig. 2A) displays a high frequency tension oscillation (range from 300 to $350 \mathrm{~Hz}$ ) overlying two beats of a lower frequency oscillation (approximately $140 \mathrm{~Hz}$ ). Neither of these oscillations has a period which corresponds to the interval between the consecutive EMG 

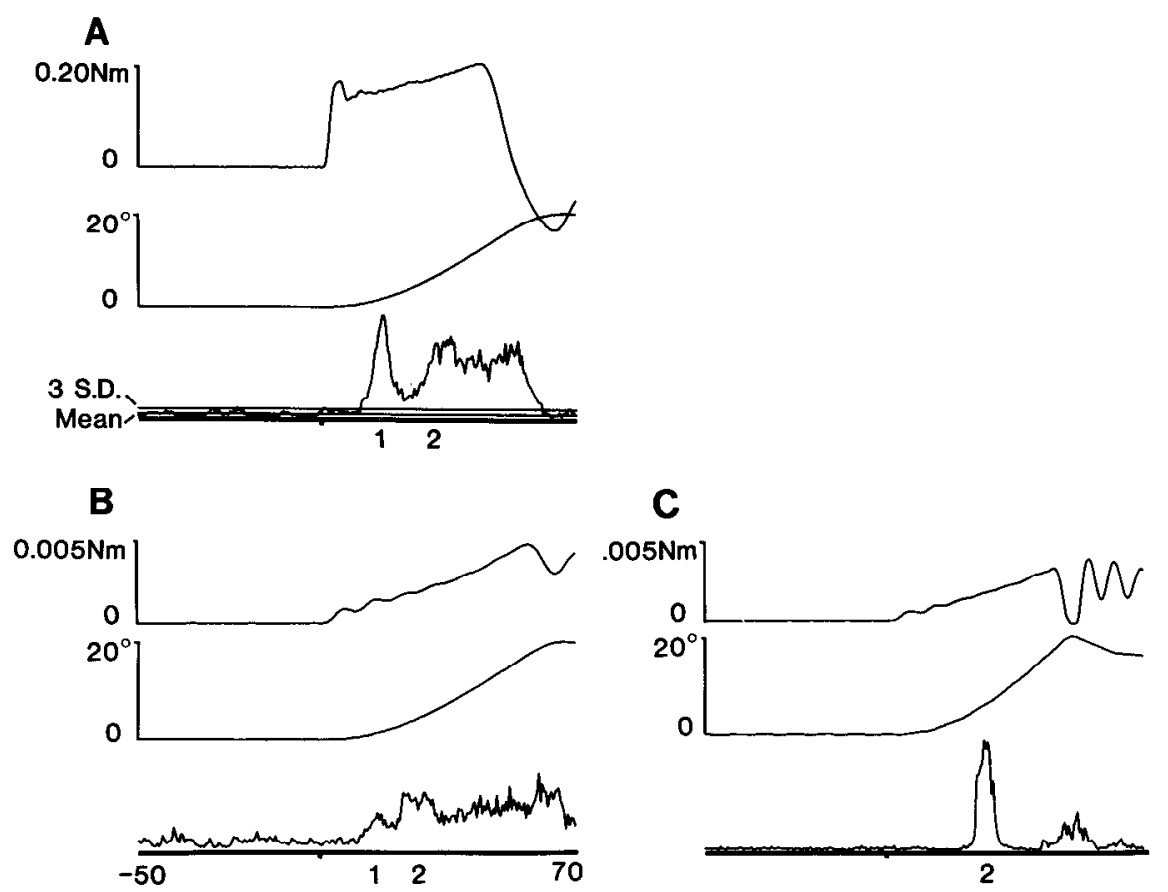

Figure 2. EMG responses in SHB $(A)$, FCU $(B)$, and FDP $(C), A, B$, and $C$ demonstrate the averaged response to torque loads extending the elbow, wrist, and MCP joint, respectively. The top traces are the average net tension for the imposed displacement shown in the middle trace. The averaged, rectified, EMG response to this displacement is shown in the bottom trace. Mean is the mean base line or tonic EMG level and $3 S D$ is the mean base line plus 3 standard deviations above base line level. The 3 SD level was used to measure the onset of EMG activity, M1 in SHB and FCU and M2 in FDP. Inasmuch as the EMG level between M1 and M2 did not fall below the $3 \mathrm{SD}$ level in SHB or FCU, the onset of M2 activity in these muscles was taken as the lowest point between the peaks. M1 and M2 components are identified by the numbers 1 and 2 under the EMG trace. The time scale is in milliseconds.

peaks. Although the two processes exhibit different frequencies, it is still possible that the EMG segmentation is related to tension oscillations in some nonlinear way (cf. Hagbarth et al., 1981) because of the complexities of the musculoskeletal system and its contact with the manipulandum.

The work of Hagbarth and co-workers (1981) makes it imperative that the investigator carefully examine the tension records, because of the possibility that tension oscillations contribute to the generation of segmented EMG responses. 'The averaged data in Figure 2 make this possibility unlikely in the case of FDP and SHB. However, given the high sensitivity of spindle primaries (Fetz et al., 1979), it could be that the averaging process obscures a variable low frequency oscillation in tension or in displacement. Therefore, the averages in Figure 3 are shown with accompanying rasters which display tension and displacement records for the individual trials used to construct the averages. The position traces in these rasters appear smooth and continuous, although very small muscle length variations are beyond the resolution of this technique. The individual tension traces in Figure 3 do not display a lower frequency component of tension oscillation. The tension oscillations in Figure $3, A$ and $B$ are similar to those seen in Figure 2, $C$ and $A$, respectively. The EMG rasters show that the EMG aclivily in individual trials does not correlate with tension oscillations in those same trials (e.g., in Fig. $3 B$ the eighth trace from the top, prominent tension oscillations are seen without accompanying EMG bursts). FCU, how- ever, did not always display such a clear dissociation between tension oscillations and EMG activity.

Hagbarth and his co-workers (1981) measured displacement both directly at the surface of the joint and from the shaft of the torque motor. They state that displacement measured from the shaft of the torque motor did not display irregularities or oscillatory variations which were evident in the displacement measured at the joint. The size of the squirrel monkeys prevented us from reliably positioning a potentiometer over the joint for measurement of displacement. However, displacement oscillations measured at the joint have been illustrated to correspond to tension oscillations (Hagbarth et al., 1981, Fig. 2). Therefore, it is unlikely that displacement oscillations, independent of tension, account for the observed EMG bursts.

Figure 2 suggests that the onset of earliest EMG response is delayed in FDP as compared with FCU and SHB. If we define M1 as a burst of EMG activity which occurs within 4 msec of the latency of the tendon tap plus isometric time constant, then M1 activity is absent in this example. It could be argued that the longer EMG onset latency is due to conduction or mechanical lags in the torque motor-joint-muscle system. These possibilities are unlikely for several reasons. First, the tendon reflex latencies in FDP and FCU are only 1 to 2 msec longer than those in SHB (see Table I). These reflexes, examples of which are shown in Figure $4 A$ for FDP and SHB, were elicited by tapping the grip of the torque motor. The oscilloscope traces in Figure $4 A$ were triggered from the 

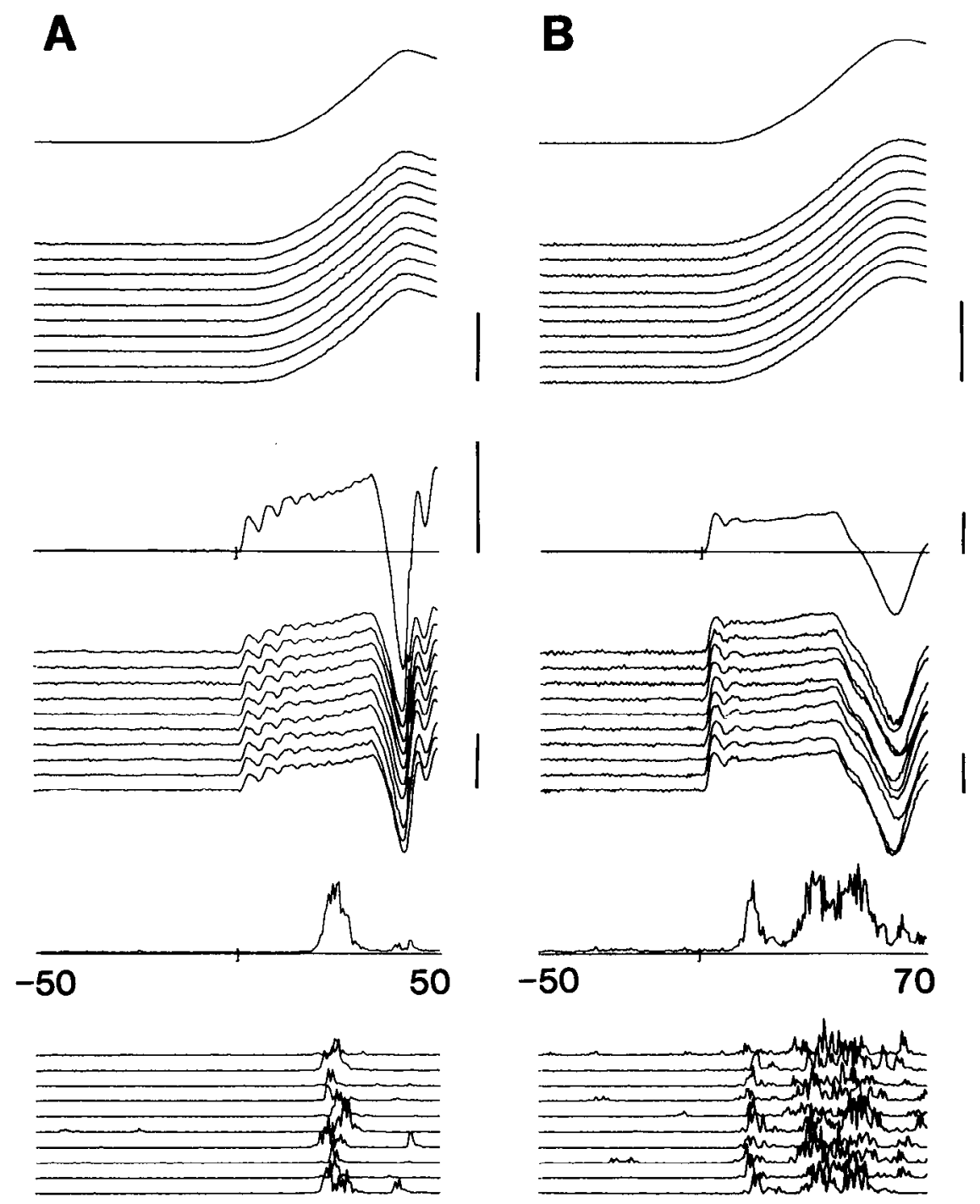

Figure 3. Rasters of displacement, tension, and EMG for FDP $(A)$ and SHB $(B)$. This figure shows a raster of the events used to construct the average trace shown above the raster. There is no evidence of discontinuity in the average displacement trace or rasters (top) of either FDP or SHB. Oscillations are present in the tension (middle) trace of FDP, but the EMG does not show an early latency peak corresponding to the first and largest component of the oscillation. A high frequency oscillation is also seen in the tension trace of SHB without evidence of EMG bursts time locked to the oscillations. There was no evidence in the tension raster of a lower frequency component corresponding to the interval between the EMG bursts found in that muscle. Position calibration bars are $20^{\circ}$ of arc in both SHB and FDP; torque calibration bars are 0.20 and $0.005 \mathrm{Nm}$ in SHB and FDP, respectively (note differences in scale of raster and average tension traces for FDP).

TABLE I

Onset latencies and durations of $M 1$ and $M 2$ activity in $S H B, F C U$, and $F D P$ of the squirrel monkey forelimb

These averages are taken from the EMG response to a maximum of six different loads in normal monkeys. Latencies are determined as described in the legend to Figure 2. The last row gives the tendon tap latencies in these same muscles (see Fig. 4).

\begin{tabular}{llll}
\hline & \multicolumn{3}{c}{ Muscle } \\
\cline { 2 - 4 } & \multicolumn{1}{c}{ FDP } & \multicolumn{1}{c}{ FCU } & SHB \\
\hline M1 onset latency & & $11 \pm 2 \mathrm{msec}$ & $10 \pm 2$ \\
M2 onset latency & $19 \pm 2$ & $19 \pm 2$ & $27 \pm 2$ \\
M2 duration & $13 \pm 2$ & $10 \pm 1$ & $15 \pm 2$ \\
& $N=37$ & $N=24$ & $N=35$ \\
Tendon reflex latency & $7.0 \pm 0.3$ & $6.9 \pm 0.2$ & $5.5 \pm 0.3$ \\
& $N=5$ & $N=6$ & $N=5$ \\
\hline
\end{tabular}

leading edge of the tension trace, and triggered from the leading edge of the tension trace, and reflex onset was measured from the EMG records. In torque motor studies, the latency of the M1 and M2 components includes the torque motor isometric time constant which is the delay between the start of square wave pulse to the torque motor and the rise in tension at the handle of the torque motor. If the isometeric time constant of the torque motor is added to the latency of the tendon jerk reflex response, then the values are within 2 to $3 \mathrm{msec}$ of the M1 latencies in Table I. Hence, delays due solely to a longer peripheral reflex pathway cannot account for the 9-msec delay in onset of EMG activity in FDP as compared with FCU and SHB. A second possible mechanism of the delay in onset of FDP EMG activity, me- 
A
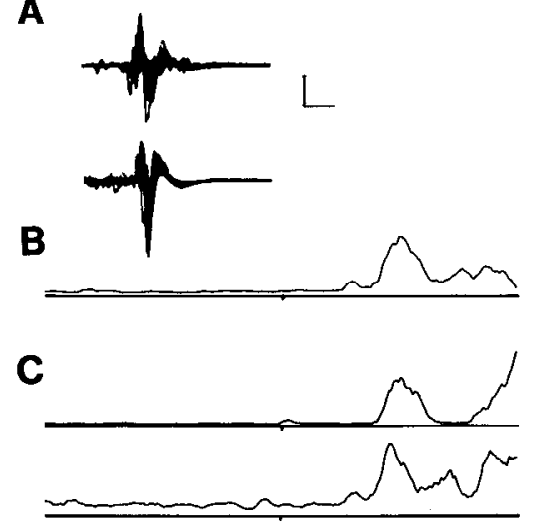

D

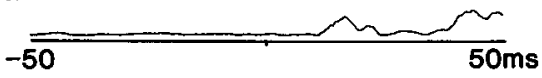

Figure 4. Early latency activity in FDP. $A$ shows the tendon jerk response in SHB (upper) and FDP (lower). Vertical and horizontal calibrations are $0.5 \mathrm{mV}$ and 5 msec, respectively. An M1 response could sometimes be discerned in the FDP EMG response of a normal monkey at usual base line levels (as in $B$ ). In some animals, no $\mathrm{M} 1$ response was seen at resting base line EMG levels (as in C', upper trace), but a M1 response became evident at higher base line levels $(C$, lower trace) for the same animal and recording session). In contrast, $\mathrm{M} 1$ activity could be seen in the absence of M2 activity in animals with the middle cerebral artery occluded (as in $D$ ).

chanical delay in the finger system as compared with the wrist and elbow systems, is discounted by observations both in normal animals and in animals with occlusion of the contralateral middle cerebral artery (Lenz et al., 1981). The data from normal animals show that an early latency EMG response can occur in some normal animals at low background EMG levels in FDP (Fig. $4 B$ ) and becomes evident at higher background EMG levels in some other normal animals (Fig. $4 C$ ). Early latency EMG activity is seen in FDP of animals with the contralateral middle cerebral artery occluded as demonstrated in Figure $4 D$. Inasmuch as M1 latency activity can occur in this muscle, one must conclude that $\mathrm{M} 1$ is frequently absent in FDP at usual background EMG levels and is not simply delayed.

Onset latencies of EMG activity in forelimb flexors. Several papers have attempted to establish that M2 onset latency varies with distance from cortex (Marsden et al., 1976b; Watt et al., 1971; Chan et al., 1979a). Although biceps is closer than flexor pollicis longus to cortex, the long latency component in human biceps occurs later than the long latency component in flexor pollicis longus (compare Figs. 3 and 11, Marsden et al., 1976b). Long latency activity in cat triceps has been found to occur at variable latency (Ghez and Shinoda, 1978; North and Tatton, 1980). Figure 5 demonstrates that the pattern of latencies in these muscles was relatively constant across a range of different velocities and in different animals. Timing of the EMG peaks was examined by measuring EMG latencies for five normal squirrel monkeys at six different initial velocities of limb displacement, as outlined under "Materials and
Methods." The latencies measured were: the onset of M1 activity, peak M1 activity, trough between $\mathrm{M} 1$ and $\mathrm{M} 2$, peak of M2 activity, and end of M2 activity. Representative EMG traces and graphs of latency measurements for SHB, FCU, and FDP are shown in Figure 5. Note that M2 activity in SHB occurs at longer latency than that found in the more distal muscles, FCU and FDP. Thus M2 activity in these distal muscles occurs at latencies during which EMG activity in SHB is at a minimum (between M1 and M2). These findings were consistent in all of the animals. Averaged onset latency data are shown in Table I along with latencies for M1 and M2 activity occurring in the proximal distal series of forearm flexors. Here again, there is a significant delay in latencies for M2 activity in biceps as compared with muscles of the forearm.

Continuing afferent input generated by ongoing joint displacement is known to contribute to the M2 peak (Vilis and Cooke, 1976) because the M2 peak is absent if the imposed joint displacement is arrested before a critical duration (Lee and Tatton, 1982). On the basis of these findings, the authors postulated that M2 activity might be generated through the combined action of a short latency pathway and a longer latency pathway. If the short latency pathway is subthreshold for an EMG response, as in FDP, then perhaps short latency mechanisms are not significant contributors to the longer latency peak. An afferent volley acting through a short latency pathway which is subthreshold for an EMG response at low base line EMG levels can give rise to a reflex response at higher base line EMG levels (Fig. $4 C$ ). However, in some animals onset of EMG activity in FDP at high base line levels approaches a minimum value which is clearly greater than tendon tap latency (Fig. $6 A$ ). In these animals there is a decrease in onset latency of FDP M2 (Fig. 6A) at increasing base line EMG levels, but it is small and parallels the decrease in FCU M1 latencies demonstrated in Figure $6 B$ (a similar decrease in latency was found at the human wrist by Bedingham, 1981). EMG onset latency (minus the torque motor isometric time constant) is plotted against base line EMG levels for FCU and FDP in Figure 6C. The line indicated by the arrow is at $7 \mathrm{msec}$, which is the tendon tap latency for these two muscles (see Table I). FCU EMG onset is seen to be asymptotic to the 7-msec line, whereas FDP is asymptotic to a line at approximately $14 \mathrm{msec}$. At these base line EMG levels and in these animals, the latency of the earliest EMG response does not approach tendon tap latencies, suggesting a relatively weak synaptic input to the motor ncurons responsible for the short latency reflex activity.

Comparison of latencies with other primate species. In order to relate latencies in normal squirrel monkeys to those in the published literature, the latency values given above were compared with the latency of reflex responses in other primate species, as shown in Table II. Where applicable, the latency measurements were corrected by the torque motor isometric time constant. The length of the peripheral conduction pathway was estimated by measuring the distance from the vertebral column to the muscle in three to eight representative animals which had been used in torque motor studies. 
$\mathbf{A}_{1}$

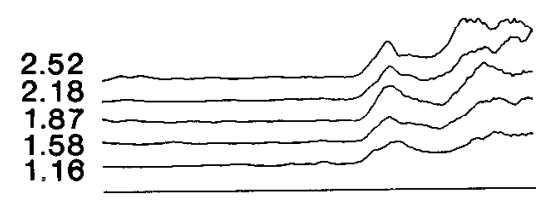

$B_{1}$

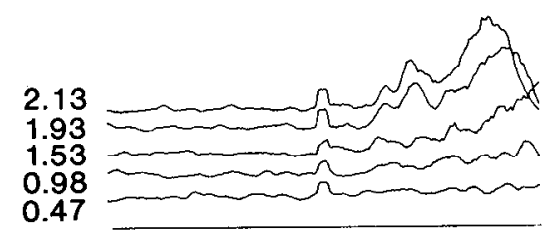

$\mathbf{C}_{1}$

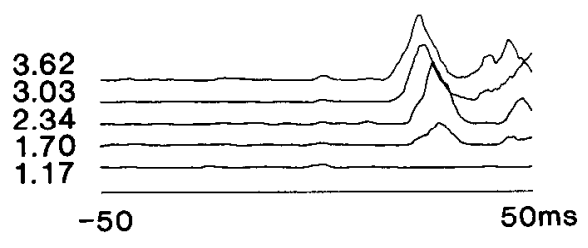

$\mathrm{A}_{2}$

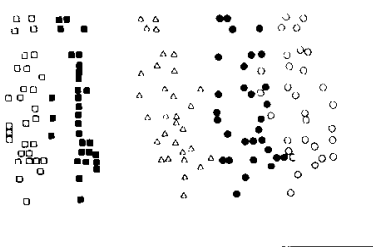

$\mathrm{B}_{2}$

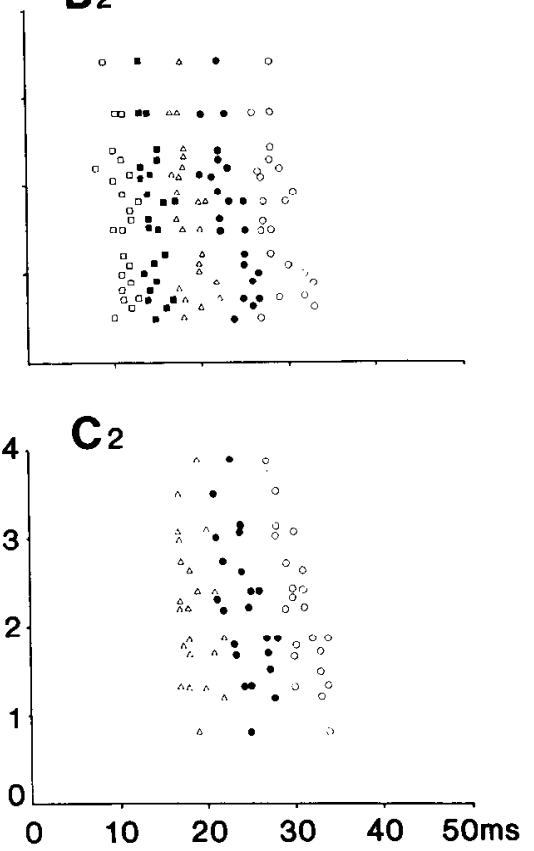

Figure 5. Relative timing of M1 and M2 activity in SHB, FCU, and FDP. The traces on the left-hand side of the figure show the EMG response in SHB $(A 1)$, FCU $(B 1)$, and FDP $(C 1)$. Note that M2 activity in SHB occurs significantly later than that in FCU and FDP. The graphs on the right-hand side plot latency measurements for M1 and M2 in SHB (A2), FCU (B2), and FDP (C2) against initial velocity. Initial velocity was measured by the displacement during the first 15 msec after the onset of the motor pulse and is given in degrees/15 msec to the left of the EMG trace. $\square$, onset M1; $\square$, peak M1; $\triangle$, trough between M1 and $\mathrm{M} 2$; - , peak of M2 activity; $\bigcirc$, end of M2 activity. Fnd of M2 activity was defined as the point at which the slope of the EMG trace returned to zero, whether M3 was present or not. Inflexions in the FCU and FDP traces at time zero are artifacts produced by the motor pulse.

Average conduction pathway length and onset latency were then plotted against each other for different species in Figure 7. Since the points for M1 and M2 peaks each present a relatively linear relation, size differences seem to account in some measure for the variation in latency between the species. Hence, the pattern of EMG response in the squirrel monkey is comparable, in terms of latency, with that found in other primate species. Note that the slopes for M1 and M2 latencies against peripheral conduction pathway for primates are different. The values for cat triceps brachii seem to correspond to thuse for primate biceps for the first peak of EMG activity but differ markedly for the second peak.

Relative size of the M1 and M2 components. Figures 2 and 4 illustrate a progressive decline in M1 activity as one examines successively more distal muscles. In order to quantify this effect the M1:(M1 + M2) ratio was calculated at several different base line EMG levels and torque loads as displayed in the average traces on the left-hand side of Figure 5. Since the M1 + M2 interval is much longer in SHB than in FCU and FDP the M1:(M1 + M2) ratio is not exactly analogous in all three muscles. Therefore, a second ratio was calculated of M1 activity to FMG activity above base line from onset of M1 to 42 msec, the average termination of M2 in biceps. An average was calculated for both of these ratios for each muscle studied, in five normal animals. These data, shown in Table III, demonstrate that M1 activity predominates over M2 activity in proximal muscles as compared with successively more distal muscles. A similar pattern has been found in the proximal-distal series of forelimb extensors (Lenz et al., 1980).

Twitch contraction time in FDP. Bawa and Tatton (1979) used single motor unit studies in muscles acting across the primate wrist to provide indirect evidence that the M2 peak is largely formed from the firing of fast 
A

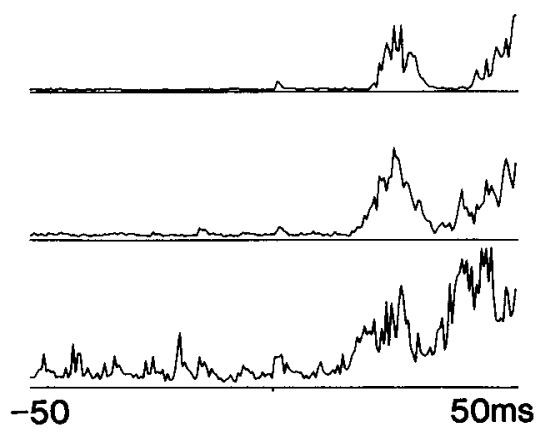

B

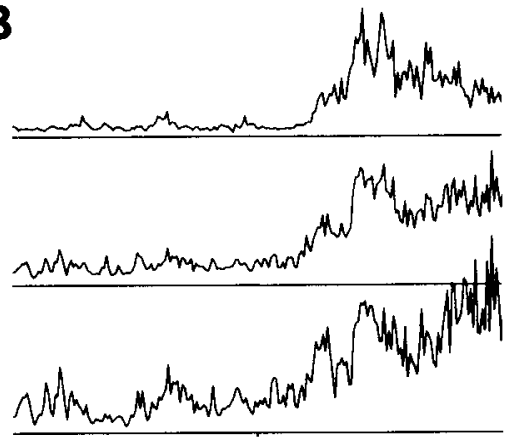

C

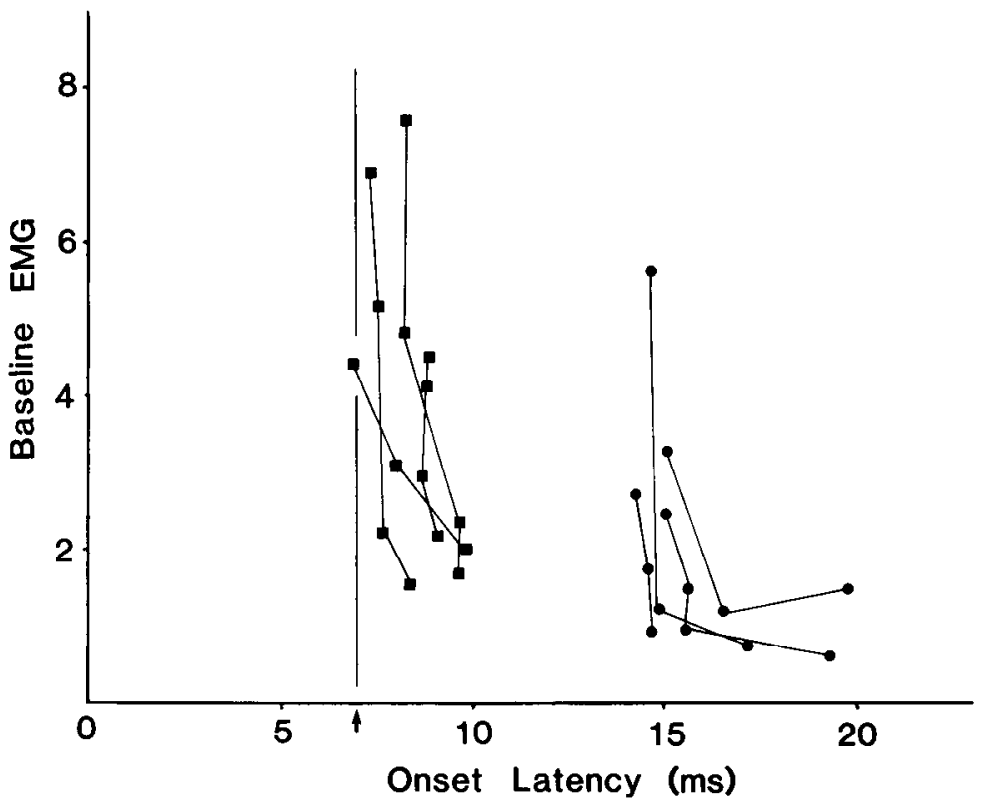

Figure 6. Latency of EMG activity at increasing base line EMG levels. The FDP reflex response at three different base line EMG levels is shown in $A$. Note that the onset latency decreases by approximately 3 msec at increasing base line levels, although an M1 component does not become apparent in this case. The FCU reflex response at three different base line EMG levels is shown in $B$. Averages extend from -50 to $50 \mathrm{msec}$ in all cases. EMG onset latency has been plotted against base line EMG levels (in $0.1 \mathrm{mV}$ ) for FDP (circles) and FCU (squares) in $C$.

twitch motor units, while the $\mathrm{M} 1$ peak results from the activity of slow twitch motor units. If it applies to the muscles of the squirrel monkey forelimb, then their finding suggests that distal muscles, such as FDP, showing predominantly $\mathrm{M} 2$ activity, contain a larger proportion of fast twitch units than muscles such as SHB whose response is composed of significant M1 and M2 responses. As an incomplete test of this hypothesis, twitch times were measured for FDP, the muscle showing the smallest M1:(M1 + M2) ratio. We were unable to reliably measure twitch times of the proximal muscles in the intact animal. The measured time to peak tension of FDP (Fig. 8) in response to a supramaximal stimulation of the ulnar and median nerves in the upper limb is 20 $\pm 3 \mathrm{msec}$, with a decay time from peak to one-half peak tension of $20 \pm 2$ msec $(n=7)$, in the intact animal. The whole muscle contraction time provides a good estimate of the tension-weighted mean contraction time (Bagust et al., 1973). Although twitch times for other muscles of the squirrel monkey forelimb have not been reported, contraction time in FDP is less than that in flexors and extensors of the macaque wrist, reported as 32 and 30 msec, respectively (Rietz and Humphrey, 1975). Furthermore, it compares well with the $19.5 \pm 0.6$ msec reported in cat flexor digitorum longus, which is largely but not exclusively composed of fast motor units (Olson and Swett, 1966; Bagust et al., 1973). Hence, it seems reasonable to conclude that FDP is composed largely of fast twitch motor units. If comparisons to the macaque are valid, then FDP is composed of proportionately more fast twitch motor units than wrist flexors and extensors, in agreement with the hypothesis of Bawa and Tatton (1979).

\section{Discussion}

The results of this study demonstrate that the EMG response to imposed joint displacement differs in proximal and distal muscles in the squirrel monkey forelimb. 
TABLE II

Onset latency and peripheral conduction pathway in biceps, triceps, and wrist flexors of several different species

Onset latencies were corrected for the torque motor isometric time constant in all cases except the results of Hammond (1954) and Chan et al. (1979a), where timing was triggered mechanically by movement. For wrist flexors (referred to as "wrist" in the table) the peripheral conduction pathway was estimated by the distance from $\mathrm{C} 7$, posterior to the medial epicondyle at the elbow and the ulnar styloid at the wrist, with the shoulder abducted to $45^{\circ}$, the elbow fully extended, and the forearm pronated. The conduction pathway for biceps was measured to the medial epicondyle with the arm in the position described above. Peripheral conduction pathway measurements were made on the human subjects or animals in the studies of Bawa and Tatton (1979), Lee and Tatton (1975), Miller and Brooks (1981), North and Tatton (1980), and the present study. Torque motor isometric time constants were measured or obtained by direct communication with the authors in these cases. In the case of other authors reporting results for studies on humans and cats, the peripheral conduction pathway and isometric time constant were assumed to be the same as the measurements taken for subjects and motors in the studies by Lee and Tatton (1975) and North and Tatton (1980).

\begin{tabular}{|c|c|c|c|c|c|}
\hline \multirow{2}{*}{ Species } & \multirow{2}{*}{ Muscle } & \multicolumn{2}{|c|}{ Onset Latency } & \multirow{2}{*}{$\begin{array}{c}\text { Conduction } \\
\text { Pathway }\end{array}$} & \multirow{2}{*}{ Author } \\
\hline & & M1 & M2 & & \\
\hline & & msec & msec & $\mathrm{cm}$ & \\
\hline \multirow[t]{3}{*}{ Human } & Biceps & 18 & 45 & 54 & Hammond (1954) \\
\hline & & 17.7 & 49.9 & & Chan et al. (1979a) \\
\hline & & 18 & 57 & & Evarts and Granit (1976) \\
\hline Cebus & Biceps & 10.5 & 35.5 & 15.5 & Miller and Brooks (1981) \\
\hline Saimiri sciureus & Biceps & 8 & 25 & 8.5 & \\
\hline \multirow[t]{2}{*}{ Cat } & Triceps & 11 & 42 & 12.5 & North and Tatton (1980) \\
\hline & Triceps & 11 & 39 & & Ghez and Shinoda (1978) \\
\hline \multirow[t]{2}{*}{ Human } & Wrist & 23 & 49 & 79 & Lee and Tatton (1975) \\
\hline & & 22.5 & 51 & & O'Rian et al. (1978) \\
\hline Macaca speciosa & Wrist & 12.5 & 32 & 42 & Bawa and Tatton (1979) \\
\hline Saimiri sciureus & FCU & 9 & 17 & 13 & \\
\hline
\end{tabular}

A

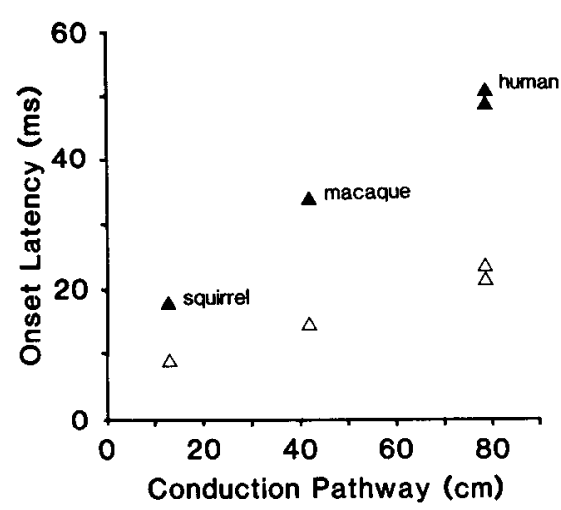

B

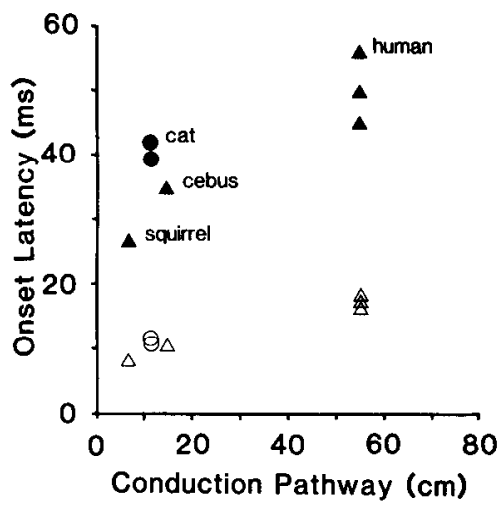

Figure 7. Relation of onset latency to the length of the peripheral conduction pathway. A displays data from wrist flexors of several different species as listed in Table II. $B$ displays the data for biceps or triceps. Open and solid triangles represent M1 and M2, respectively. Circles represent the first and second peaks in cat triceps. The species is designated below the point representing the $\mathrm{M} 2$ or second peak in each case.

TABLE III

$M 1:(M 1+M 2)$ ratios for $F D P, F C U$, and $S H B$

M1 and M2 latencies were determined as described in the legend to Figure 2. The second column gives the ratio of M1 activity in a muscle to activity occurring in that muscle over the M1 + M2 interval of SHB. The area of an EMG peak was defined as the area under the peak above base line.

\begin{tabular}{ccc}
\hline Muscle & M1:M1 + M2 & M1:SHB M1 + M2 \\
\hline FDP & $0.10 \pm 0.11$ & $0.08 \pm 0.10$ \\
FCU & $0.38 \pm 0.08$ & $0.23 \pm 0.09$ \\
SHB & $0.42 \pm 0.09$ & $0.42 \pm 0.09$ \\
\hline
\end{tabular}

At low base line FMG levels M1 activity is pronounced in SHB, less pronounced in FCU, and not discernible in FDP of normal animals. The M1 and M2 bursts of EMG activity are not time locked to tension oscillations initi- ated by the imposed torque pulse. The M2 peak in biceps occurs later than the M2 peaks in FCU and FDP, raising the possibility that M2 may not be generated by the same mechanism in the different muscles.

The multiple bursts of EMG activity which occur in response to joint displacement have recently been attributed to oscillations in tension following the onset of the torque pulse (Hagbarth et al., 1981). Since tension oscillations were observed in the present study, great care was taken to exlude them as the sole cause of EMG bursts. This was achieved by increasing the mechanical damping or varying the resonant frequency of the system so that tension oscillations were not appropriately timed to explain the observed bursts of EMG activity. Although EMG bursts may be produced by repetitive activation of the same short latency reflex pathway (Hagbarth et al., 
1981), the present study demonstrates that EMG bursts may also be generated by other mechanisms, independent of tension oscillations.

The variation in reflex responses in the different upper limb muscles is consistent with previous reports. Marsden (Marsden et al., 1976a) has studied the EMG response to perturbations interrupting flexion of flexor pollicis longus. The early latency response is virtually absent in this muscle. In contrast to this result, a clear M1 component is reported in human wrist flexors (Lee and Tatton, 1978), biceps (Hammond 1954, 1960; Evarts and Granit 1976; Marsden et al., 1976b; Chan et al., 1979a) and in nonhuman primate wrist flexors and extensors (Tatton et al., 1978; Bawa and Tatton, 1979) and biceps (Evarts and Tanji, 1976; Vilis and Cooke, 1976; Cooke and Eastman, 1977). Therefore, there is support for the idea that a progressive decrease in early latency activity occurs as one proceeds distally in the primate upper limb.

The differences in the response between muscles extend to latency of peaks as well as to the relative mag-

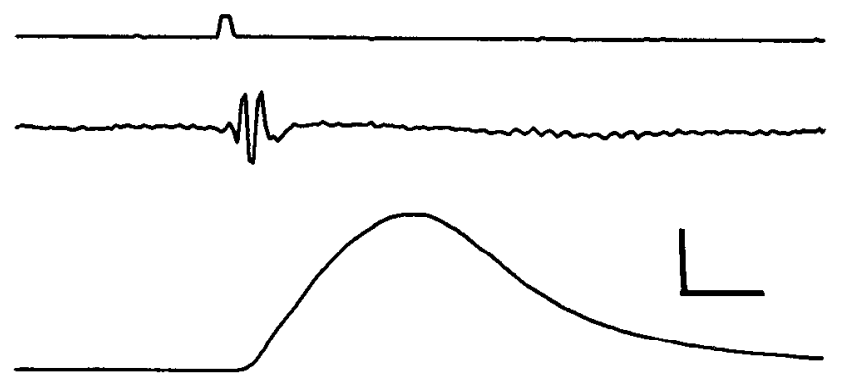

Figure 8. Twitch contraction for FDP. This figure shows the tension response in FDP to supramaximal stimulation of the nerve. From top to bottom, the traces show the time of the peripheral nerve stimulus, the EMG response, and the tension response, respectively. Vertical and horizontal calibration bars are $20 \mathrm{gm}$ and $10 \mathrm{msec}$. nitudes of M1 and M2 activity. The onset of M2 activity in SHB occurs later than that in FCU and FDP, the reverse of what would be expected if the onset of M2 activity were dependent on central conduction delay in all muscles studied. The latencies observed in squirrel monkey are consistent with those found in other primate species and with the result, from Marsden's work, that human long latency activity occurs later in biceps than in flexor pollicis longus (Marsden et al., 1976b). North and Tatton (1980) have suggested that the silent period preceding the second peak in cat triceps (labeled M 2 by Ghez and Shinoda (1978)) is due to an inhibition or disfacilitation following the first peak. A similar phenomenon may cause the relative delay in squirrel monkey SHB M2 because the "trough" between M1 and M2 in biceps is usually very pronounced (Fig. 2A) compared with FCU (Figs. $2 B$ and $5 B 1$ ), although the EMG level never fell below base line over this interval as was reported for cat triceps (North and Tatton, 1980). Finally, it is important to emphasize that central conduction delay alone cannot necessarily be assumed to account for the relative onset latency of $\mathrm{M} 2$ activity throughout the primate forelimb.

Comparison of reflex responses between monkeys and humans is of particular importance in the choice of animals for experimental models of human disease (Lee and Tatton, 1975; Tatton et al., 1979; Lenz el al., 1981). Onset latencies of M1 and M2 peaks in different primate species seem to vary almost linearly with the length of the peripheral conduction pathway, suggesting that reflexes are comparable in these species. On this same basis, the long latency component in the cat may not be analogous to that described in primate species (Fig. 7).

Bawa and Tatton (1979) have shown that M1, M2, and M3 components at the primate wrist are largely formed by the firing of distinct populations of single motor units. On the basis of recruitment order to maintained loads and median interspike interval, they suggested that slow

TABLE IV

Motor unit composition of muscles in proximal-distal sequence

The species and muscle studied and the percentage of slow and fast fibers are indicated.

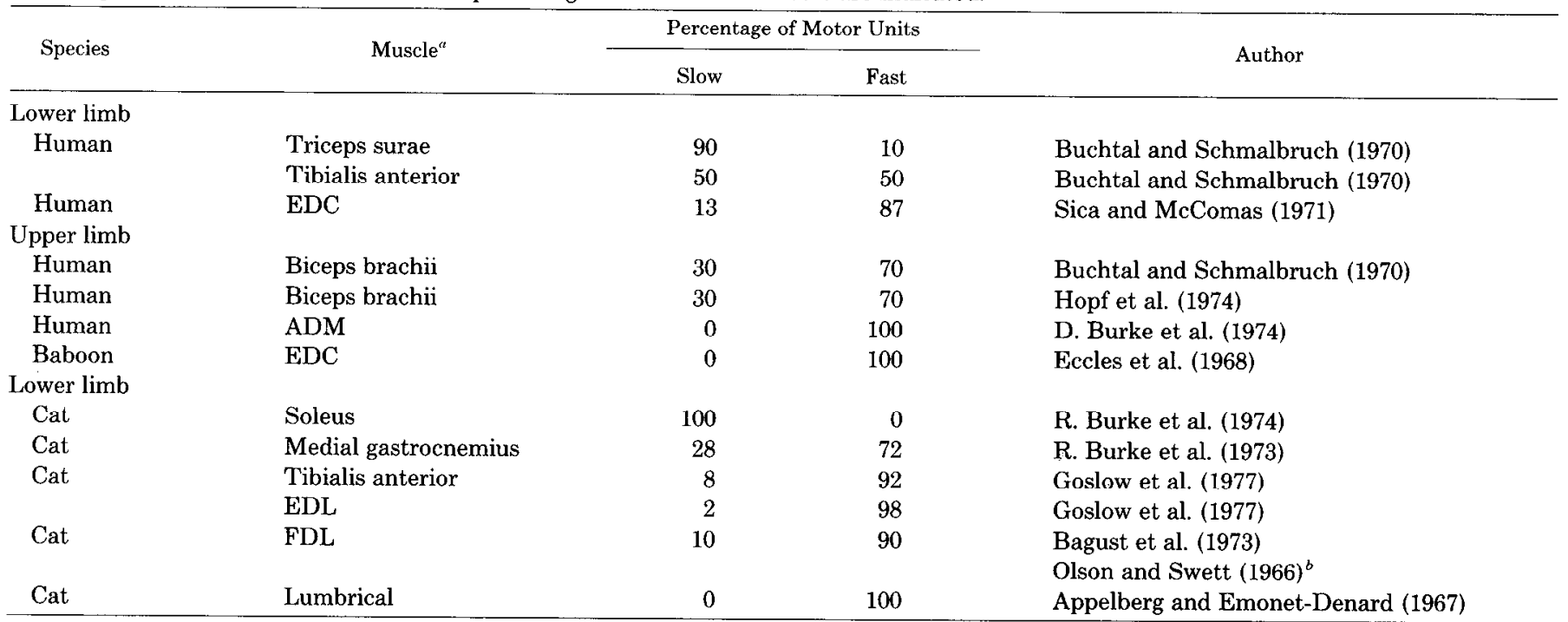

${ }^{a}$ EDC, extensor digitorum communis; $\Lambda \mathrm{Dm}$, adductor digiti minimi; EDl, extensor digitorum longus; FDL, flexur digilorum longus.

${ }^{b}$ Olson and Swett (1966) report fast and slow twitch motor unit populations in cat FDL. Bagust et al. (1966) do not identify fast and slow twitch motor unit populations, although the distribution of units by contraction time is similar to that of Olson and Swett (1966). 
twitch motor units contributed to M1 response and fast twitch motor units contributed to $\mathrm{M} 2$ and $\mathrm{M} 3$ responses. Physiological studies of motor units comprising different muscles in the cat hindlimb and in humans suggest (see Table IV) that distal muscles have a higher proportion of fast twitch fibers than do proximal and postural muscles. In nonhuman primates, the only physiological study of single motor units found that extensor digitorum communis in the baboon is exclusively composed of fast twitch motor units (Eccles et al., 1968). Hence, it is possible that the M1:M1 + M2 ratio may to some extent reflect the relative proportion of slow twitch motor units in the muscle studied. Such a proposal is consistent with the findings reported here, that the response is often limited to long latency activity in fast muscles, such as squirrel monkey FDP (Fig. 8).

Imposed displacements exceeding a critical duration have been shown to be essential for the generation of the M2 peak (Lee and Tatton, 1982), whereas the M1 peak can be evoked with displacements too short to result in M2 activity (Vilis and Cooke, 1976). One possible explanation of these results is that continuing afferent input acting through a short latency pathway is required to facilitate a longer latency mechanism (Lee and Tatton, 1982). A substantial M1 response, which must involve a short latency pathway, was found in the muscles studied in both of these reports. The relatively small or absent M1 component in the FDP EMG response to MCP joint displacement suggests that the short latency pathway is less effective in recruiting motor neurons innervating this muscle. In some animals it was so weak that the EMG onset was not asymptotic to tendon tap latencies at high base line EMG levels (Fig. 6). Therefore, the role of short latency pathways in the generation of FDP M2 activity is almost certainly less than in more proximal muscles.

This study demonstrates that there are substantial differences between the relative contribution of short and long latency mechanisms to the reflex activation of motoneurons in distal as compared with proximal upper limb flexor muscles in the primate. Therefore, the results of studies of the mechanisms underlying the generation of reflex activity in one muscle group in a given species cannot necessarily be assumed to be identical for another muscle group or species. Finally, the dysfunction of mechanisms which contribute to the EMG response to imposed joint displacement may be more evident in one muscle group than in another. The significance of the muscle group studied may be reflected in the differences between studies of EMG responses in the human thumb after cortical lesions (Marsden et al., 1977) and those in the cat forelimb after decerebration (Ghez and Shinoda, 1978).

\section{References}

Appelburg, B., and F. Emonet-Denard (1967) Motor units of the first superficial lumbrical muscle of the cat. J. Neurophysiol. 30: 154-160.

Bagust, J., S. Knott, D. M. Lewis, J. C. Luck, and R. A. Westerman (1973) Isometric contractions of motor units in a fast twitch muscle of the cat. J. Physiol. (Lond.) 231: 87-104.

Basmajian, J. V., and G. Stecko (1962) A new bipolar electrode for electromyography. J. Appl. Physiol. 17: 849-850.

Bawa, P., and W. G. Tatton (1979) Motor unit responses in muscles stretched by imposed displacements of the monkey wrist. Exp. Brain Res. 37: 417-438.

Bedingham, W. (1981) Input-output properties of the human wrist reflex-A model for studying neuro-motor diseases. M.Sc. thesis, University of Toronto.

Brown, T. H. I., P. M. H. Rack, and H. F. Ross (1976) Sinusoidal driving of the finger joints. J. Physiol. (Lond.) 263: 184-185P.

Buchtal, F., and H. Schmalbruch (1970) Contraction times and fiber types in intact human muscle. Acta Physiol. Scand. 79: 435-452.

Buchtal, F., and H. Schmalbruch (1980) Motor unit of mammalian muscle. Physiol. Rev. 60: 90-142.

Burke, D., N. Skuse, and A. K. Lethlean (1974) Isometric contraction of abductor digiti minimi muscle in man. J. Neurol. Neurosurg. Psychiatry 37: 825-834.

Burke, R. E., D. N. Levine, P. Tsairsis, and F. E. Zajac (1973) Physiologic types and histochemical properties of motor units of the cat gastrocnemius. J. Physiol. (Lond.) 234: 723-748.

Burke, R. E., D. N. Levine, M. Salcman, and P. Tsairsis (1974) Motor units of the cat soleus muscle: Physiological, histochemical, and morphological characteristics. J. Physiol. (Lond.) 238: 503-514.

Chan, C. W. Y., G. Melvill-Jones, R. E. Kearney, and D. G. D. Watt (1979a) The "late" electromyographic response to limb displacement in man. 1. Evidence for a supraspinal contribution. Electroencephalogr. Clin. Neurophysiol. 46: 173-181.

Chan, C. W. Y., R. E. Kearney, and G. Melvill-Jones (1979b) Tibialis anterior response to sudden ankle displacements in normal and parkinsonian subjects. Brain Res. 173: 303-314.

Colbourne, T. R., and E. V. Evarts (1978) Use of brushless DC torque motors in studies of neuromuscular function. Prog. Clin. Neurophysiol. 4: 153-166.

Cooke, J. D., and M. J. Eastman (1977) Long loop reflexes in the tranquilized monkey. Exp. Brain Res. 27: 491-500.

Eccles, R. M., C. G. Phillips, and W. Chien-ping (1968) Motor innervation, motor unit organization and afferent innervation of the $\mathrm{m}$. extensor digitorum communis of the baboon's forearm. J. Physiol. (Lond.) 198: 179-192.

Evarts, E. V., and R. Granit (1976) Relations of reflexes and intended movements. Brain Res. 44: 1-14.

Evarts, E. V., and J. Tanji (1976) Reflex and intended responses in motor cortex pyramidal tract neurons of monkey. J. Neurophysiol. 39: 1069-1080.

Evarts, E. V., and W. J. Vaughn (1978) Intended arm movements in response to externally produced arm movements. Prog. Clin. Neurophysiol. 4: 178-192.

Fetz, E. E., E. Jankowska, T. Johannisson, and J. Lipski (1979) Autogenetic inhibition of motoneurons by impulses in group 1 A muscle afferents. J. Physiol. (Lond.) 293: 173-195.

Ghez, C., and Y. Shinoda (1978) Spinal mechanisms of the functional stretch reflex. Exp. Brain Res. 32: 55-68.

Goslow, G. E., W. E. Cameron, and D. G. Stuart (1977) The fast twitch motor units of cat ankle flexors. 1. Tripartite classification on basis of fatigability. Brain Res. 134: 35-46.

Gottlieb, G. L., and C. G. Agarwal (1979) Response to sudden torques about the ankle in man: Myotatic reflex. J. Neurophysiol. 42: 91-105.

Hagbarth, K. E., J. V. Hagglund, E. U. Wallin, and R. R. Young (1981) Grouped spindle and electromyographic responses to abrupt wrist extension movements in man. J. Physiol. (Lond.) 312: 81-96.

Hammond, P. H. (1954) Involuntary activity in biceps following the sudden application of velocity to the abducted forearm. J. Physiol. (Lond.) 127: 17-18P.

Hammond, P. H. (1960) An experimental study of servo action in human muscular control. In Proceedings of the Third International Conference in Medical Electronics, pp. 190199, Institute of Electronic Engineering, London.

Hopf, H. C., R. L. Herbort, M. Gnass, H. Gunther, and K. Lowitzsch (1974) Fast and slow contraction times associated 
with fast and slow spike conduction of skeletal muscle fibers in normal subjects and in spastic hemiparesis. Z. Neurol. 206: 193-202.

Lee, R. G., and W. G. Tatton (1975) Motor responses to sudden limb displacements in primates with specific CNS lesions and in human patients with motor system disorders. Can. J. Neurol. Sci. 2: 285-293.

Lee, R. G., and W. G. Tatton (1978) Long loop reflexes in humans: Clinical application. Prog. Clin. Neurophysiol. 4: 334-341.

Lee, R. G., and W. G. Tatton (1982) Long latency reflexes to imposed displacements of the human wrist: Dependence on duration of movement. Exp. Brain Res. 45: 207-216.

Lenz, F. A., R. R. Tasker, I. C. Bruce, and W. G. Tatton (1980) The EMG response to joint displacement in flexors and extensors of the squirrel monkey forelimb. Can. Physiol. 11: 98.

Lenz, F. A., R. R. Tasker, and T. Tsuda (1981) MCA occlusion in the squirrel monkey: Clinical picture and torque motor studies. Neurology (Minneap.) 30: 49.

Magladery, J. W., R. D. Teasdale, A. M. Park, and W. E. Porter (1951) Electrophysiological studies of nerve and reflex activity in normal man. V. Excitation and inhibition of two neuron reflexes by afferent impulses in the same trunk. Bull. Johns Hopkins Hosp. 88: 520-537.

Marsden, C. D., P. A. Merton, and H. B. Morton (1976a) Servo action in the human thumb. J. Physiol. (Lond.) 257: 1-44.

Marsden, C. D., P. A. Merton, and H. B. Morton (1976b) Stretch reflex and servo action in a variety of human muscles. J. Physiol. (Lond.) 259: 531-560.

Marsden, C. D., P. A. Merton, H. B. Morton, and J. Adam (1977) The effect of lesions of sensorimotor cortex and capsular pathways on servo responses from the human long thumb flexor. Brain 100: 503-526.

Miller, A. D., and V. B. Brooks (1981) Late muscular responses to arm perturbations persist during supraspinal dysfunctions in monkeys. Exp. Brain Res. 41: 146-158.

Milner-Brown, H. S., R. B. Stein, and R. G. Lee (1975) Synchronization of human motor units: Possible roles of exercise and supraspinal reflexes. Electroencephalogr. Clin. Neurophysiol. 38: 245-254.

Mortimer, J. A., and M. J. Johnson (1976) Modification in monosynaptic and long loop reflexes by movement tasks and preloads. In Proceedings of the International Symposium on Human Reflexes and Motor Disorders, J. E. Desmedt, ed., pp. 131-132, Brain Research Unit, University of Brussels.

North, A. E. G., and W. G. Tatton (1980) Temporal relations between motor cortical and EMG responses to imposed forelimb displacements in the cat. Soc. Neurosci. Abstr. 7: 392.

Olson, C. B., and C. P. Swett (1966) A functional and histochemical characterization of motor units in a heterogeneous muscle (flexor digitorum longus) of the cat. J. Comp. Neurol. 128: 475-498.

O'Riain, M. D., R. D. G. Blair, and J. T. Murphy (1978) The influence of visual cues on muscle stretch reflexes. Can. J. Physiol. Pharmacol. 56: 19-22.

Rack, P. M. H., H. F. Ross, and T. I. P. Brown (1978) Reflex responses during sinusoidal movements of the human limbs. Prog. Clin. Neurophysiol. 4: 216-228.

Rietz, R., and D. R. Humphrey (1975) Dynamic properties of rhesus monkey forelimb muscles. Soc. Neurosci. Abstr. 2: 185 .

Sica, R. E. P., and A. J. McComas (1971) Fast and slow twitch fibers in a human muscle. J. Neurol. Neurosurg. Psychiatry 34: 113-120.

Stanley, E. F. (1978) Reflexes evoked in human thenar muscles during voluntary activity and their conduction pathways. J. Neurol. Neurosurg. Psychiatry 41: 1016-1023.

Tatton, W. G., and P. Bawa (1979) Input-output properties of motor unit responses in muscles stretched by imposed displacement of the monkey wrist. Exp. Brain Res. 37: 439-457.

Tatton, W. G., S. D. Forner, G. L. Gerstein, W. W. Chambers, and E. W. Lui (1975) The effect of post-central cortical lesions on motor responses to sudden upper limb displacements in monkeys. Brain Res. 196: 108-113.

Tatton, W. G., P. Bawa, I. C. Bruce, and R. G. Lee (1978) Longloop reflexes in monkeys: An interpretive base for human reflexes. Prog. Clin. Neurophysiol. 4: 229-245.

Tatton, W. G., P. Bawa, and I. C. Bruce (1979) Altered motor cortical activity in motor cortical rigidity. Adv. Neurol. 10: 141-160.

Upton, A. R. M., A. J. McComas, and R. E. P. Sica (1971) Potentiation of "late" responses evoked in muscles during effort. J. Neurol. Neurosurg. Psychiatry 34: 699-711.

Vilis, T. R., and J. D. Cooke (1976) Modulation of the functional stretch reflex by the segmental reflex pathway. Exp. Brain Res. 25: 247-254.

Watt, D. G. D., C. W. Y. Chan, and G. Melville-Jones (1971) Is the late response to muscle stretch in man mediated through a long-loop reflex pathway? DRB Aviat. Med. Res. Unit. Rep. 3: 210-222.

Weisendanger, M. (1978) Comments on the problem of transcortical reflexes. J. Physiol. (Paris) 74: 325-330. 